\title{
Coplanar-Waveguide Ultra-Wideband Bandpass Filter Based on Interdigital Structure
}

\author{
Yanyan Peng and Feng Xu \\ Department of Electronic Science and Engineering \\ Nanjing university of Posts and Telecommunication \\ Nanjing, China \\ feng.xu@njupt.edu.cn
}

\begin{abstract}
In this paper, a novel ultra wideband (UWB) bandpass filter based on interdigital structure is proposed. The proposed UWB bandpass filter is designed on coplanar waveguide (CPW) with the passband obtained by interdigital structure on the step-change center strip of CPW. The rectangular curling slots are adopted to improve the stopband characteristics and the normal slots are used to restrain the insertion loss of the filter in passband. The interdigital structure and all slots are on the same side of a circuit board which makes the UWB bandpass filter compact and easily realized. Simulations and measurements verify the design idea and the proposed filter has a more than $150 \%$-3dB fractional bandwidth centered at $5.56 \mathrm{GHz}$ as well as sharp skirt selectivity at the upper passband edge.
\end{abstract}

Keywords-Bandpass filter; coplanar waveguide; defected ground structure; interdigital coupling; ultra wideband

\section{INTRODUCTION}

It has been known that bandpass filters with small size and light weight are fundamental components of high-speed wireless-communications systems. Interdigital bandpass filter have attracted wide attentions and extensive studies [1-3] with the advantage of compact, easy integration, low insertion loss, and wide fractional bandwidth. While many filters are implemented on microstrip, three structures are usually used for realizing CPW UWB filters: cascading CPW lowpass and bandpass structures [4-5], hybrid microstrip-CPW structure [68] and multiple-mode resonators [9-10]. There is few reported research work has successfully developed on UWB CPW bandpass filter based on interdigital coupling structure. In [11], a novel CPW interdigital filter is optimized by short circuited quarter wave split strips resonator. However bonding wires to connect the split strips of the resonator make it difficult to process and integrate. The UWB bandpass filter which uses interdigital capacitor coupling to generate additional transmission poles is mentioned in [12] and achieves $110 \%$ passband.

In this paper, a novel CPW UWB bandpass filter based on interdigital structure is introduced. The size of the bandpass filter which occupies only half one guided wavelength at 5.56 $\mathrm{GHz}$ is $2 \mathrm{~cm} \times 3 \mathrm{~cm} \times 0.635 \mathrm{~cm}$, and it is more compact than the filter size in [12]. Moreover, the fractional band width of the filter at center frequency reaches $150 \%$ and it has a steeper stopband. Most important, since the whole design of the filter is on the same side of a circuit board, the proposed filter will be easier to process and integrate. As regards the entire structure of the proposed filter, we first introduce a middle widened step-change on the center strip of CPW which contributes to the cutoff at high frequency end as well as broadband coupling of interdigital structure. Furthermore, interdigital capacitance instead of gap capacitance is obtained to realize wideband coupling. The interdigital structure is scheduled to suppress low frequency but not ideal for making stopband at high frequency end. Finally, a significant improvement of the characteristics of the filter is obtained by adding disturbance slots: rectangular curling slots used to get steep attenuation and wide stopband and normal slots used to suppress the passband insertion loss. In section 2 the sizes and effect of interdigital structures and disturbance slots are investigated and discussed. As with the disturbance slots, they are symmetrically etched on the both sides of the center strip which makes the designed filter compact, simple process and easy integration. By optimizing the position and dimension of the slots, an excellent UWB filter of the CPW version is fulfilled. At last, it is worth to note that the filter has been fabricated and measured to verify the idea and design procedure.

\section{FILTER DESIGN}

A CPW version UWB filter based on interdigital structure is illustrated in Fig. 1. It is realized on a single layer metallic substrate (Rogers RT/duroid 6010 (tm)), with relative dielectric constant of 10.2 and thickness of $0.635 \mathrm{~mm}$. The sizes of the UWB filter are listed in Table I and optimized by simulation software. The size of center strip and slot are chosen to match with 50 ohms for measure convenient.

TABLE I.
\begin{tabular}{|c|c|c|c|c|c|c|c|c|c|}
\hline Maker & a & b & c & d & g1 & g2 & f0 & f & j \\
\hline Length/mm & 30 & 20 & 1.4 & 2.2 & 0.9 & 0.6 & 0.1 & 0.2 & 1.1 \\
\hline
\end{tabular}

Middle widened center strip of CPW will causes a cutoff at high frequency end, in this work we assign the cutoff zone around $12 \mathrm{GHz}$. On the other hand the middle widened center strip enlarges the coupling width of interdigital structure. 
From the simulation curves in Fig. 2 and Fig. 3, out-ofband performance is not very good. Etched slot on ground which is known as defected ground structure (DGS) is usually used to improve the filter performance.

Early in 2008, Sun used the rectangular curling slots as one of the interdigital coupling arm[13],which proved to be functional in bring out more transmission zeros and wider stopband. Besides, its normalized transmission zero versus the normalized stub length has been discussed in detail. Different from the reference mentioned, our work obtains the rectangular curling slots as DGS on the CPW ground and provides an additional degree of freedom in adjusting the distribution of extra transmission zeros by adjusting the location of slots.

Optimized physical sizes and location of the slots are illustrated in Fig. 4. Fig. 5 shows the significant effect of rectangular curling slot on out-of-band performance improvement at the high frequency end. The role of the normal slots is also clearly displayed in suppressing insertion loss. The $8.53 \mathrm{GHz}$ passband with insertion loss of only $0.32 \mathrm{~dB}$ as well as $4.5 \mathrm{GHz}-15 \mathrm{~dB}$ stopband and sharp skirt selectivity near the stopband are obtained. Additionally, all the slots are symmetrically etched at the both sides of the input/output CPW center strip.

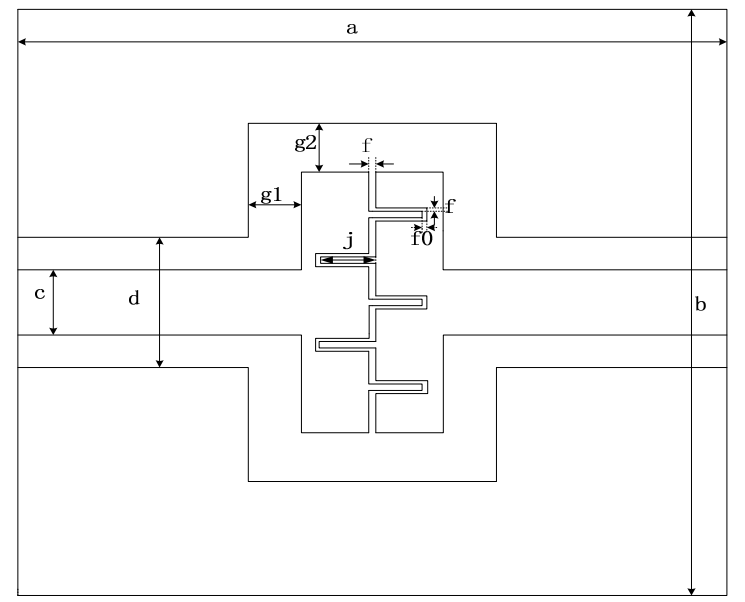

Fig. 1 Physical structures of a CPW version UWB filter based on interdigital structure.

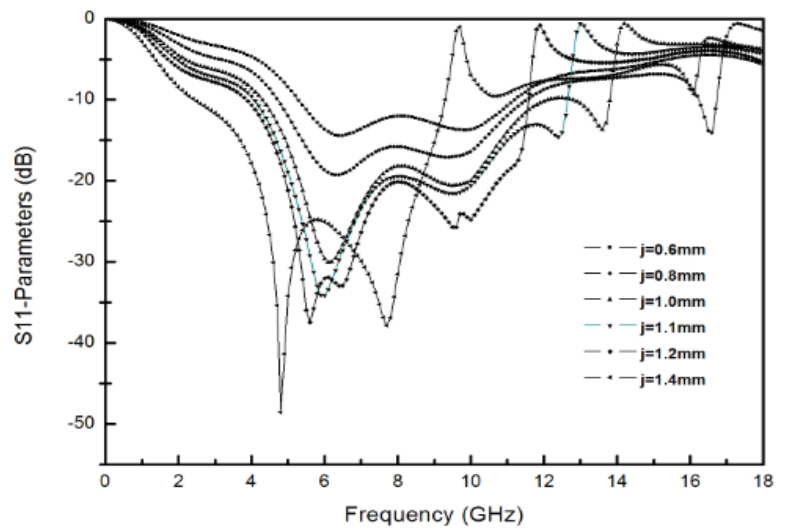

Fig. 2 Simulated S11 parameters of the filter with different interdigital depth.
It is obvious that the proposed filter bears merits such as good performance, concise procedure, easy fabrication and integration, compared to the reference [4-12].

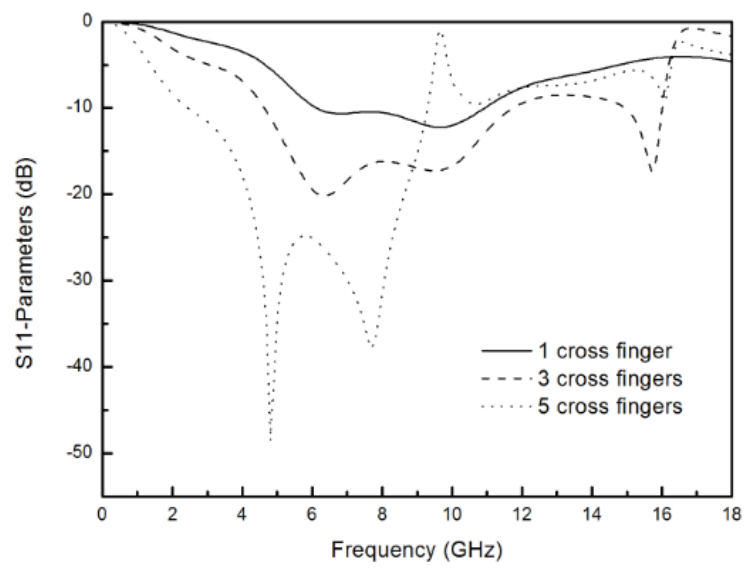

Fig. 3 Simulated S11-parameters of the filter with different number of the cross fingers.

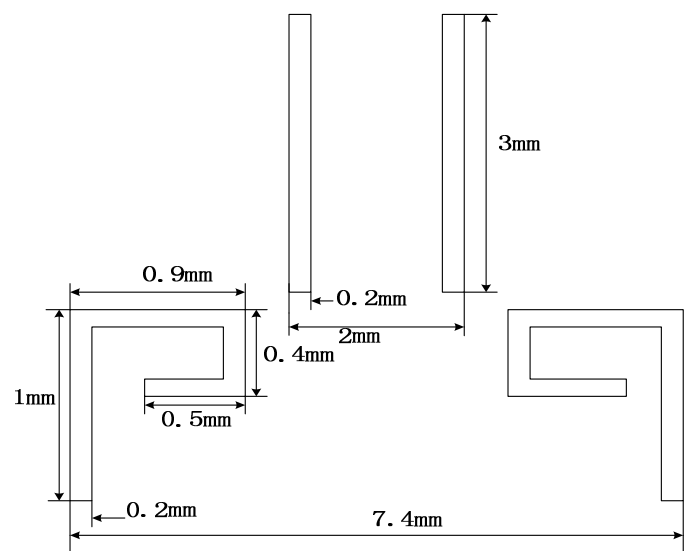

Fig. 4 Physical sizes of the slots.

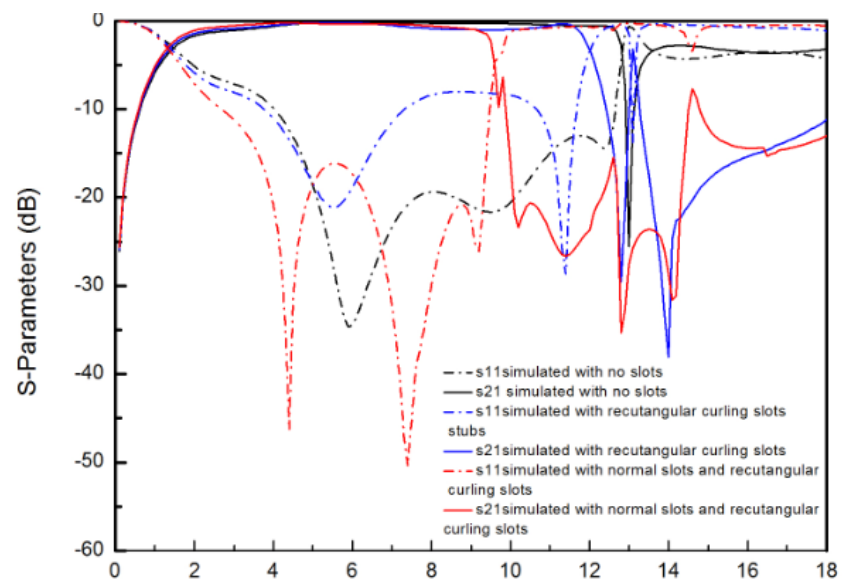

Fig. 5 Simulated S-parameters of the filter with normal slots and rectangular curling slots.

\section{MEASUREMENT}

After investigating the effects of these parameters, we can design a CPW version UWB filter. A filter with a fractional bandwidth $(-3 \mathrm{~dB})$ of more than $150 \%$ at the center frequency 5.56 $\mathrm{GHz}$ and $4.5 \mathrm{GHz}-15 \mathrm{~dB}$ stopband from $9.9 \mathrm{GHz}$ to 14.4 
$\mathrm{GHz}$ has been realized. To verify the design, this filter has been fabricated and its photograph is shown in Fig. 6. Measurements have been realized by Vector Network Analyzer Agilent. A specialized test fixture (Anritsu MODEL $3680 \mathrm{~K})$ is used to combine the designed filter with Vector Network Analyzer which can avoid additional reflection loss of the welding SMA connector. The comparison between the simulated and measured results of the filter is illustrated in Fig. 7. The good agreement between the simulated results and the measured data can be found.

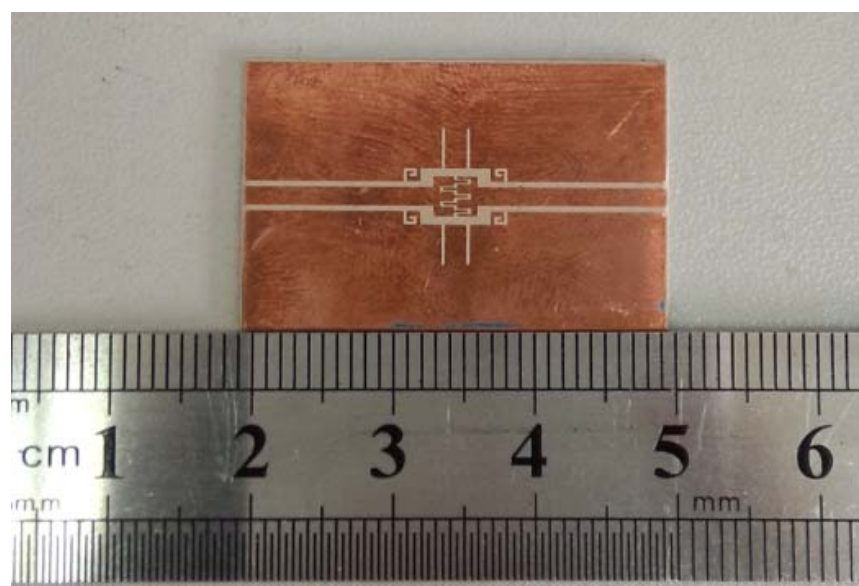

Fig. 6 Photo of the fabricated CPW filter with the interdigital structure and slots.

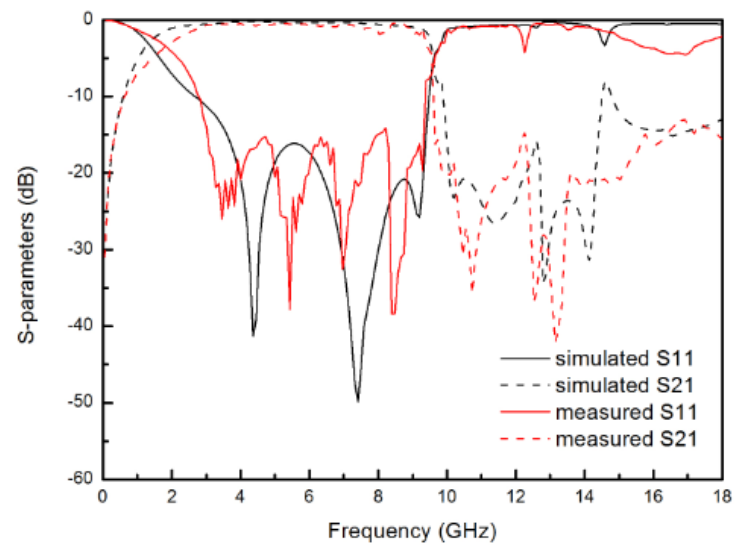

Fig. 7 Simulated and measured S-parameters of the UWB filter.

\section{CONCLUSION}

A novel CPW UWB bandpass filter based on interdigital structure is proposed and verified. The etched rectangular curling slots and normal slots on the ground of CPW are used to improve the performance of the filter. The proposed filter has the excellent characteristics, such as the compact size, ultra wide passband, steep skirt selectivity at the high frequency end of passband, and wide stopband. In addition to these excellent characteristics this filter is easier to process and integrate. Therefore, this CPW filter promises for the application in UWB wireless systems.

\section{ACKNOWLEDGMENT}

This work was supported by the specially appointed professor program foundation of Jiangsu province of China, the national natural science foundation of China under grant No. 61171052, the doctoral fund of ministry of education of China under grant No. 20123223110004 and the fund of state key laboratory of millimeter waves of China under grant No. K201414.

\section{REFERENCES}

[1] S. Sun and L. Zhu, "Capacitive-Ended Interdigital Coupled lines for UWB bandpass filters with improved out-of-band performances”, IEEE Microw. Wireless Compon. Lett., vol. 16, no. 8, pp. 440-442, Aug. 2006.

[2] Y. Mu, Z. Ma and D. Xu "A novel compact interdigital bandpass filter using multiplayer cross-coupled folded quarter-wavelength resonators", IEEE Microw. Wireless Compon. Lett.,vol. 15, no. 12, pp.847 -849, 2005.

[3] Z. Wang, F. Nasri and C. W. Park, "Compact tri-band notched UWB bandpass filter based on interdigital hairpin finger structure”, Proc. IEEE Wireless Microw. Tech. Conf., pp.1 -4, 2011.

[4] Y.S. Lin, W.C. Ku, C.H. Wang, and C.H. Chen, "Wideband coplanarwaveguide bandpass filters with good stopband rejection”, IEEE Microwave Wireless Compon. Lett,vol. 14 no.9,pp. 422-424,2004.

[5] N. W. Chen and K. Z. Fang, "An ultra-broadband coplanar-waveguide bandpass filter with sharp skirt selectivity”, IEEE Microw. Wireless Compon. Lett., vol. 17, no. 2, pp.124 -126 2007.

[6] K. Li, D. Kurita, and T. Matsui, "An ultra-wideband bandpassfilter using broadside-coupled microstrip-coplanar waveguide structure”, IEEE MTT-S Int. Dig., pp. 675-678, Jun. 2005.

[7] T. N. Kuo, S. C. Lin, and C. H. Chen, "Compact ultra-wideband bandpass filter using composite microstrip-coplanar-waveguide structure", IEEE Trans. Microwave Theory Tech., vol. 54, pp. 37723778, Oct. 2006.

[8] J.-W. Baik, T.-H.Lee, and Y.-S. Kim, "UWB bandpass filter using microstrip to CPW transition with broadband balun”, IEEE Microw. Wireless Compon. Lett., vol. 17, no. 12, pp. 846-848, Dec. 2007.

[9] J. Gao, L. Zhu, W. Menzel, and F. Bögelsack, "Short-circuited CPW multiple-modere sonator for ultra-wideband (UWB)bandpass filter", IEEEMicrow.Wireless Compon. Lett., vol. 16, no. 3, pp. 104-106,Mar. 2006.

[10] F. Aryanfar and K. Sarabandi, "Compact millimeter-wave filters using distributed capacitively loaded CPW resonators," IEEE Trans. Microw.Theory Tech., vol. 54, no. 3, pp. 1161-1165, Mar. 2006.

[11] C. Y. Chang and D. C. Niu, "A novel CPW interdigital filter," AsiaPacific Microw. Conf. Dig., pp. 621-624, Dec. 2001.

[12] J. Gao, L. Zhu, W. Menzel, and F. Bögelsack, "Ultra-Wideband Bandpass Filter on Coplanar Waveguide: Proposal and Implementation” International Journal of RF and Microwave Computer-Aided Engineering, vol.17,no. 2, pp.225-235,Mar.2007.

[13] S. Sun, L. Zhu and H.-H.Tan, "A compact wideband bandpass filter using transversal resonator and asymmetrical interdigital coupled lines”, IEEE Microw. Wireless Compon. Lett., vol. 18, no. 3, pp.173 175, 2008. 